\title{
THE EXISTENCE QUESTION IN THE CALCULUS OF VARIATIONS: A DENSITY RESULT
}

\author{
ARRIGO CELLINA AND CARLO MARICONDA
}

(Communicated by Barbara Lee Keyfitz)

\begin{abstract}
We show the existence of a dense subset $\mathscr{D}$ of $\mathscr{C}(\mathbb{R})$ such that, for $g$ in it, the problem

$$
\text { minimum } \int_{0}^{T} g(x(t)) d t+\int_{0}^{T} h\left(x^{\prime}(t)\right) d t, x(0)=a, x(T)=b
$$

admits a solution for every lower semicontinuous $h$ satisfying growth conditions
\end{abstract}

\section{INTRODUCTION}

The first basic aim of the Calculus of Variations is the problem of yielding sufficient conditions for the existence of solutions to the classical minimum problem

$$
\text { Minimize } \int_{0}^{T} f\left(t, x(t), x^{\prime}(t)\right) d t: x(0)=a, x(T)=b
$$

on a suitable space of functions $x$.The traditional answer to the sufficiency has been to impose that the map $x^{\prime} \mapsto f\left(t, x, x^{\prime}\right)$ be convex (see [Ce]); more recently, in an effort to provide existence criteria other than convexity in $x^{\prime}$, some special sufficient condition has been given. More precisely, for the class of maps of the form $f\left(t, x, x^{\prime}\right)=g(t, x)+h\left(t, x^{\prime}\right)$, existence of solutions has been obtained by requiring that the map $x \mapsto g(t, x)$ be monotonic (for $x$ in $\mathbb{R})[\mathrm{M}]$ or, for $x$ in $\mathbb{R}^{n}$, that the same map be concave [C-C]. Hence, for maps of the form $g(x)+h\left(x^{\prime}\right)$ the property of yielding existence of solutions so far seems to belong to a very narrow and special class of functions: those that are either convex in $x^{\prime}$ or concave or monotonic in $x$. Existence seems to be a property related to very special geometric behaviour in $x$ or in $x^{\prime}$. The purpose of this note is to show that (for $x$ in $\mathbb{R}$ ) this is not so. We consider the class of functions $g(x)+h\left(x^{\prime}\right)$ where $g: \mathbb{R} \rightarrow \mathbb{R}$ is continuous and $h$ is lower semicontinuous; we show that there exists a subset $\mathscr{D}$ of the space of continuous functions, dense for the topology of uniform convergence

Received by the editors November 4, 1991 and, in revised form, July 20, 1992.

1991 Mathematics Subject Classification. Primary 49A05; Secondary 28B05.

Key words and phrases. Calculus of variations, subdifferential, relaxed problem, bipolar, Liapunov, measurable partition, equi-integrability. 
on compacta, such that, for $g$ in $\mathscr{D}$, problem $(\mathrm{P})$ has existence of solutions for every function $h$ satisfying the usual growth conditions.

\section{NOTATION AND PRELIMINARY RESULTS}

Let us denote by $\partial f(x)$ the subdifferential of a function $f$ at $x$. Also, by $\operatorname{extr}(S)$ we mean the set of extreme points of $S$. The following lemma will be used later.

Lemma 1. Let $f: \mathbb{R} \rightarrow \mathbb{R}$ be convex, l.s.c., $f\left(x^{\prime}\right) \geq \psi\left(\left|x^{\prime}\right|\right)+\gamma \quad(\gamma \in \mathbb{R}$, $\psi \geq 0$ convex, l.s.c. and $\left.\lim _{r \rightarrow \infty} \psi(r) / r=+\infty\right)$. Then, for each measurable $x^{\prime}: I=[\alpha, \beta] \rightarrow \mathbb{R}$ with the property that $t \mapsto f\left(x^{\prime}(t)\right) \in L^{1}$, there exists an integrable selection $\omega$ of $\Phi(t): t \mapsto(\partial f)^{-1}\left(\partial f\left(x^{\prime}(t)\right)\right)$ with values in $\operatorname{extr}(\Phi(t))$ and such that

$$
\int_{I} \omega(t) d t=\int_{I} x^{\prime}(t) d t, \quad \int_{I} f(\omega(t)) d t=\int_{I} f\left(x^{\prime}(t)\right) d t
$$

and, for each $t$,

$$
\int_{\alpha}^{t} \omega(s) d s \leq \int_{\alpha}^{t} x^{\prime}(s) d s
$$

Proof. Let us first remark that each inverse image under $\partial f$ of a point $c \in \mathbb{R}$ is closed, convex ( $f$ being convex), and bounded, since $\lim _{x^{\prime} \rightarrow \infty} f\left(x^{\prime}\right) /\left|x^{\prime}\right|=$ $+\infty$. It follows that each $(\partial f)^{-1}(c)$ is either empty or a closed interval. Let $\left(c_{i}\right)_{i \in J \subset \mathbb{N}}$ be the values of $\partial f$ whose inverse image under $\partial f$ is a nontrivial interval $\left[a_{i}, b_{i}\right]\left(a_{i} \neq b_{i}\right)$, and set $K_{i}=\left(x^{\prime}\right)^{-1}\left(\left[a_{i}, b_{i}\right]\right)$ for $i$ in $J, K_{0}=$ $I \backslash \bigcup_{i} K_{i}$. Then, by Lemma 3.4 in [A-C], there exists a measurable selection $\sigma_{i}$ of the set-valued map $\Phi(t) \chi_{K_{i}}(t)$ with values in $\operatorname{extr}\left(\Phi(t) \chi_{K_{i}}(t)\right)=\left\{a_{i}, b_{i}\right\}$ such that

$$
\int_{I} \sigma_{i}(t) \chi_{K_{i}}(t) d t=\int_{I} x^{\prime}(t) \chi_{K_{i}}(t) d t
$$

and, for each $t$,

$$
\int_{\alpha}^{t} \sigma_{i}(s) \chi_{K_{i}}(s) d s \leq \int_{\alpha}^{t} x^{\prime}(s) \chi_{K_{i}}(s) d s .
$$

For each $i \in J$, we have

$$
\int_{I} f\left(x^{\prime}(t)\right) \chi_{K_{i}}(t) d t=\int_{I} f\left(a_{i}\right)+c_{i}\left(x^{\prime}(t)-a_{i}\right) \chi_{K_{i}}(t) d t
$$

hence, by (1)

$$
\begin{aligned}
\int_{I} f\left(x^{\prime}(t)\right) \chi_{K_{i}}(t) d t & =\int_{I} f\left(a_{i}\right)+c_{i}\left(\sigma_{i}(t)-a_{i}\right) \chi_{K_{i}}(t) d t \\
& =\int_{I} f\left(\sigma_{i}(t)\right) \chi_{K_{i}}(t) d t
\end{aligned}
$$

since $\sigma_{i}(t) \in\left\{a_{i}, b_{i}\right\}$. It follows that, for each $n \in \mathbb{N}$,

$$
\int_{I} \psi\left(\left|x^{\prime}\right| \chi_{K_{0}}+\sum_{i \leq n}\left|\sigma_{i}\right| \chi_{K_{i}}\right) d t \leq \int_{I} f\left(x^{\prime}(t)\right) d t-\int_{I} \gamma d t
$$


i.e., the functions $\omega_{n}=x^{\prime} \chi_{K_{0}}+\sum_{i<n} \sigma_{i} \chi_{K_{i}}, n \in \mathbb{N}$, are equi-integrable. Set $\omega(t)=x^{\prime} \chi_{K_{0}}(t)+\sum_{i} \sigma_{i}(t) \chi_{K_{i}}(t)=\lim _{n} \omega_{n}(t)$.Vitali's convergence theorem [E-T, VIII, Corollary 1.3] yields that $\omega$ is in $L^{1}$. By (1), (2), (3), $\omega$ has the required properties.

Definition. $\mathscr{D}$ is the subset of $\mathscr{C}(\mathbb{R})$ of those piecewise affine functions $g$ such that: (i) any interval in which $g$ is affine with a given nonzero slope is bounded; (ii) adjacent to any such maximal interval, on both the left and the right, is one in which $g$ is constant; and (iii) any bounded set contains only finitely many such intervals.

Proposition 1. $\mathscr{D}$ is dense in $\mathscr{C}(\mathbb{R})$.

\section{MAIN RESULT}

Consider the following problem:

Problem P.

$$
\text { Minimize } \int_{0}^{T} g(x(t)) d t+\int_{0}^{T} h\left(x^{\prime}(t)\right) d t
$$

on the subset of $W^{1, p}([0, T], \mathbb{R})$ of those functions satisfying the boundary conditions $x(0)=a, x(T)=b$.

We shall consider the following

Growth condition G. If $p=1$, there exist a convex 1.s.c. function $\psi$ such that $\lim _{r \rightarrow \infty} \psi(r) / r=+\infty, \gamma \in \mathbb{R}$, and $h\left(x^{\prime}\right) \geq \psi\left(\left|x^{\prime}\right|\right)+\gamma ;$ if $p>1$, there exist $\sigma>0(\beta / \sigma$ being strictly less than the best Sobolev constant), and $h\left(x^{\prime}\right) \geq \sigma\left|x^{\prime}\right|^{p}+\gamma$.

We have the following

Theorem 1. Let $p \geq 1$ and $g \in \mathscr{D}$ be such that $g(x) \geq \alpha-\beta|x|^{p}$ for every $x$ $(\alpha, \beta \in \mathbb{R})$. Let $h$ satisfy the growth condition $(\mathrm{G})$. Then, Problem $(\mathrm{P})$ admits at least a solution.

Proof. Let $\tilde{x}$ be a solution to the relaxed problem associated to (P), and set

$$
\Delta_{1}=\min \{\tilde{x}(t): t \in[0, T]\}, \quad \Delta_{2}=\max \{\tilde{x}(t): t \in[0, T]\} .
$$

Let $d_{1}$ be the greater discontinuity point of $g^{\prime}$ less than or equal to $\Delta_{1}, d_{2}<$ $\cdots<d_{n-1}$ be those inside $]-\Delta_{1}, \Delta_{2}\left[, d_{n}\right.$ be the next after $d_{n-1}$, and set

$$
\epsilon=\frac{1}{5} \min \left\{\left|d_{i+\mathrm{i}}-d_{i}\right|: i=1, \ldots, n-1\right\} .
$$

Claim (a). We claim that $[0, T]$ can be partitioned in a countable union of disjoint intervals $I_{j}(j \in \mathbb{N})$ such that $g$ is monotonic on $\widetilde{x}\left(I_{j}\right)$.

Proof of the claim. Consider the three sets $A, V, B$ defined by

$$
\begin{gathered}
\left.A=\bigcup_{i=1}^{n-1}\right] d_{i}+\epsilon, d_{i+1}-\epsilon\left[, \quad V=\left\{d_{i}-\epsilon, d_{i}+\epsilon: i=1, \ldots, n\right\}\right. \\
\left.B=\bigcup_{i=1}^{n}\right] d_{i}-2 \epsilon, d_{i}+2 \epsilon[.
\end{gathered}
$$

By the continuity of $\tilde{x}$, the inverse image of $A$ under $\tilde{x}$ is a countable union of disjoint relatively open subintervals $\left(\sigma_{i}, \tau_{i}\right)$ of $[0, T]$. The image of each subinterval is contained in one of the open intervals $] d_{i}+\epsilon, d_{i+1}-\epsilon$ [ on which 
$g$ is affine. By the continuity of $\tilde{x}$, there exists $\delta$ such that $|t-s|<\delta \Rightarrow$ $|\tilde{x}(t)-\tilde{x}(s)|<\epsilon$. Consider those subintervals $\left(\sigma_{i}, \tau_{i}\right)$ whose diameter $\tau_{i}-\sigma_{i} \geq$ $\delta$, say for $i=1, \ldots, m$. These are the first elements of our partition. Again by continuity, for each $i$, at least one between $\widetilde{x}\left(\sigma_{i}\right)$ and $\widetilde{x}\left(\tau_{i}\right)$ is in $V$ (actually both, except for the case $\sigma_{i}=0$ or $\tau_{i}=T$ ). Consider the finite union of closed subintervals of $[0, T]$ that is the complement of the finite union of open subintervals $\left(\sigma_{i}, \tau_{i}\right), i=1, \ldots, m$; they are the intervals $\left[0, \sigma_{1}\right], \ldots$, $\left[\tau_{m-1}, \sigma_{m}\right],\left[\tau_{m}, T\right]$. For $t$ in this complement, $\widetilde{x}(t)$ is in $B$. In fact, either $t$ is in $\tilde{x}^{-1}(A) \backslash \bigcup_{i=1}^{m}\left(\sigma_{i}, \tau_{i}\right)$ or $\tilde{x}(t)$ is in $\bigcup_{i}\left[d_{i}-\epsilon, d_{i}+\epsilon\right]$.

In the first case, from the choice of $\delta$ and the remark on the behaviour at the extremes of the intervals, there exists $d_{i}$ such that $\left|\tilde{x}(t)-d_{i}\right|<2 \epsilon$, i.e., $\tilde{x}(t) \in B$.

The second case holds since each $\left[d_{i}-\epsilon, d_{i}+\epsilon\right]$ is in $B$. Since $B$ is open, its counter image $\tilde{x}^{-1}(B)$ is a countable collection of open subintervals. Consider the image of any such subinterval: it is contained in one (and only one) ]$d_{i}-2 \epsilon, d_{i}+2 \epsilon$ [. On one of $] d_{i}-2 \epsilon, d_{i}$ [ or $] d_{i}, d_{i}+2 \epsilon$ [ $g$ is constant, on the other affine; $g$ is monotonic on $] d_{i}-2 \epsilon, d_{i}+2 \epsilon$ [. Intersect each subinterval with the finite collection of intervals $\left[\tau_{i}, \sigma_{i+1}\right]: i=0, \ldots, m$. The union of this countable collection of intervals and of $\left(\sigma_{i}, \tau_{i}\right): i=1, \ldots, m$ is the required partition of $[0, T]$.

Claim (b). By [E-T, Proposition IX.3.1] in the case $p>1$ and by a slight modification of the same reasoning in the case $p=1$ under the "superlinearity" growth condition $(\mathrm{G})$, there exist measurable $p_{1}, p_{2}, v_{1}, v_{2}:[0, T] \rightarrow \mathbb{R}\left(p_{i} \geq\right.$ $\left.0, p_{1}+p_{2}=1\right)$ such that

$$
\left\{\begin{array}{l}
\tilde{x}^{\prime}=p_{1} v_{1}+p_{2} v_{2} \quad \text { a.e. } \\
h^{* *}\left(\tilde{x}^{\prime}(t)\right)=p_{1}(t) h\left(v_{1}(t)\right)+p_{2}(t) h\left(v_{2}(t)\right) \quad \text { a.e. }
\end{array}\right.
$$

hence, $\operatorname{extr}\left(\partial h^{* *}\right)^{-1}\left(\partial h^{* *}\left(\tilde{x}^{\prime}(t)\right)\right)=\left\{v_{1}(t), v_{2}(t)\right\}$ a.e. Let $I_{j}=\left(\alpha_{j}, \beta_{j}\right)$ be one of the intervals considered in (a). Let us explicitly carry out the construction for the case $g^{\prime}<0$ on $] d_{i-1}, d_{i}[$; the other cases are treated similarly. We claim that there exists a measurable partition $E_{1}^{j}, E_{2}^{j}$ of $\left[\alpha_{j}, \beta_{j}\right]$ such that:

(i) $v_{1} \chi_{E_{1}^{j}}+v_{2} \chi_{E_{2}^{j}} \in L^{p}\left(\alpha_{j}, \beta_{j}\right)$;

(ii) $\int_{\alpha_{j}}^{\beta_{j}} v_{1} \chi_{E_{1}^{j}}+v_{2} \chi_{E_{2}^{j}} d t=\int_{\alpha_{j}}^{\beta_{j}} p_{1} v_{1}+p_{2} v_{2} d t$;

(iii) $\int_{\alpha_{j}}^{t} v_{1} \chi_{E_{1}^{j}}+v_{2} \chi_{E_{2}^{j}} d s \geq \int_{\alpha_{j}}^{t} p_{1} v_{1}+p_{2} v_{2} d s$ for each $t \in\left(\alpha_{j}, \beta_{j}\right)$;

(iv) $\int_{\alpha_{j}}^{\beta_{j}} p_{1}(t) h\left(v_{1}(t)\right)+p_{2}(t) h\left(v_{2}(t)\right) d t$

$$
=\int_{\alpha_{j}}^{\beta_{j}} h\left(v_{1}(t)\right) \chi_{E_{1}^{j}}(t)+h\left(v_{2}(t)\right) \chi_{E_{2}^{j}}(t) d t
$$

(v) $\left|\int_{\alpha_{j}}^{t} v_{1} \chi_{E_{1}^{j}}+v_{2} \chi_{E_{2}^{j}} d s-\int_{\alpha_{j}}^{t} p_{1} v_{1}+p_{2} v_{2} d s\right|<\epsilon$ for each $t \in\left(\alpha_{j}, \beta_{j}\right)$.

Proof of the claim. Set $\psi\left(x^{\prime}\right)=\left|x^{\prime}\right|^{p}$ if $p>1$. Let us first remark that by La Valleee-Poussin's Theorem, the set

$$
\mathscr{H}=\left\{f \in L^{1}\left(\alpha_{j}, \beta_{j}\right): \int_{\alpha_{j}}^{\beta_{j}} \psi(|f|) \leq \int_{\alpha_{j}}^{\beta_{j}} h^{* *}\left(\tilde{x}^{\prime}(t)\right) d t-\int_{\alpha_{j}}^{\beta_{j}} \gamma d t\right\}
$$

is equi-integrable. Let $\rho>0$ be such that $\int_{A}|f| d t<\epsilon / 2$ for each measurable subset $A$ of $I_{j}$ whose measure is less than $\rho$ and for each $f \in \mathscr{H}$. Let $\alpha_{j}=$ $\gamma_{0}<\gamma_{1}<\cdots<\gamma_{m}=\beta_{j}$ be a subdivision of $I_{j}$ such that $\max _{i}\left|\gamma_{i+1}-\gamma_{i}\right|<\rho$. By Lemma 1 and the above remark on the inverse image of the subdifferential of 
$h^{* *}$, for each interval $\left[\gamma_{k}, \gamma_{k+1}\right]$, there exists a measurable partition $E_{1, k}, E_{2, k}$ satisfying:

(i) $v_{k} \quad v_{1} \chi_{E_{1, k}}+v_{2} \chi_{E_{2, k}} \in L^{p}\left(\gamma_{k}, \gamma_{k+1}\right)$;

(ii) $\int_{k} \quad \int_{\gamma_{k}}^{\gamma_{k+1}} v_{1} \chi_{E_{1, k}}+v_{2} \chi_{E_{2, k}} d t=\int_{\gamma_{k}}^{\gamma_{k+1}} p_{1} v_{1}+p_{2} v_{2} d t$;

(iii) $)_{k} \quad \int_{\gamma_{k}}^{t} v_{1} \chi_{E_{1, k}}+v_{2} \chi_{E_{2, k}} d s \geq \int_{\gamma_{k}}^{t} p_{1} v_{1}+p_{2} v_{2} d s$ for each $t \in\left(\gamma_{k}, \gamma_{k+1}\right)$;

(iv) $)_{k} \quad \int_{\gamma_{k}}^{y_{k+1}} \sum_{i=1}^{2} p_{i}(t) h\left(v_{i}(t)\right) d t=\int_{\gamma_{k}}^{\gamma_{k+1}} \sum_{i=1}^{2} h\left(v_{i}(t)\right) \chi_{E_{i, k}}(t) d t$.

Set $E_{1}^{j}=\bigcup_{k=0}^{m-1} E_{1, k}, E_{2}^{j}=\bigcup_{k=0}^{m-1} E_{2, k}$. Then (i), (ii), (iii), (iv) can be trivially deduced from their corresponding $(\mathrm{i})_{k},(\mathrm{ii})_{k},(\mathrm{iii})_{k},(\mathrm{iv})_{k}$. In order to prove (v), fix $t \in\left(\alpha_{j}, \beta_{j}\right)$ and let $k$ be such that $t \in\left[\gamma_{k}, \gamma_{k+1}[\right.$. Let us write that

$$
\int_{\alpha_{j}}^{t} \sum_{i}\left(p_{i}-\chi_{E_{i}^{j}}\right) v_{i} d s=\int_{\alpha_{j}}^{\gamma_{k}} \sum_{i}\left(p_{i}-\chi_{E_{i}^{j}}\right) v_{i} d t+\int_{\gamma_{k}}^{t} \sum_{i}\left(p_{i}-\chi_{E_{i}^{j}}\right) v_{i} d s .
$$

By $(\mathrm{ii})_{k}$, the first term of the right-hand side of the above equality is zero. Furthermore, we have

$$
\psi\left(\left|v_{1}\right| \chi_{E_{1}^{j}}+\left|v_{2}\right| \chi_{E_{2}^{j}}\right) \leq h\left(v_{1}(t)\right) \chi_{E_{1}^{j}}(t)+h\left(v_{2}(t)\right) \chi_{E_{2}^{j}}(t)-\gamma
$$

so that, by (iv) $)_{k}, v_{1} \chi_{E_{1}^{j}}+v_{2} \chi_{E_{2}^{j}} \in \mathscr{H}$. Let us recall that $\left|t-\gamma_{k}\right|<\rho$; hence, by equi-integrability we have

$$
\begin{aligned}
\left|\int_{\alpha_{j}}^{t} \sum_{i}\left(p_{i}-\chi_{E_{i}^{j}}\right) v_{i} d s\right| & \leq \int_{\gamma_{k}}^{t} p_{1}\left|v_{1}\right|+p_{2}\left|v_{2}\right| d s+\int_{\gamma_{k}}^{t}\left|v_{1}\right| \chi_{E_{1}^{j}}+\left|v_{2}\right| \chi_{E_{2}^{j}} d s \\
& <\frac{\epsilon}{2}+\frac{\epsilon}{2}=\epsilon,
\end{aligned}
$$

which proves the claim.

Claim (c). Let us denote by $\bar{x}_{j}:\left[\alpha_{j}, \beta_{j}\right]=I_{j} \rightarrow \mathbb{R}$ the function defined by

$$
\bar{x}_{j}(t)=\tilde{x}\left(\alpha_{j}\right)+\int_{\alpha_{j}}^{t} v_{1} \chi_{E_{1}^{j}}+v_{2} \chi_{E_{2}^{j}} d s .
$$

Then, by (i), $\bar{x}_{j}^{\prime} \in L^{p}\left(\alpha_{j}, \beta_{j}\right)$, and by (ii) we have $\bar{x}_{j}\left(\beta_{j}\right)=\tilde{x}\left(\beta_{j}\right)$. Furthermore, by (iv),

$$
\int_{\alpha_{j}}^{\beta_{j}} h^{* *}\left(\widetilde{x}^{\prime}(t)\right) d t=\int_{\alpha_{j}}^{\beta_{j}} h\left(\bar{x}_{j}^{\prime}(t)\right) d t .
$$

Since, by definition, $\bar{x}_{j}\left(\alpha_{j}\right)=\tilde{x}\left(\alpha_{j}\right)$ then, by (iii), $\bar{x}_{j}(t) \geq \tilde{x}(t)$ for every $t \in I_{j}$. Moreover, by $(\mathrm{v}),\left|\bar{x}_{j}(t)-\widetilde{x}(t)\right|<\epsilon$ for every $t \in I_{j}$; whence, $\bar{x}_{j}(t) \epsilon$ ] $d_{i}-3 \epsilon, d_{i}+3 \epsilon$ [. Then, for $g$ nonincreasing on the above interval,

$$
g\left(\bar{x}_{j}(t)\right) \leq g(\widetilde{x}(t)) \quad \text { for every } t \in I_{j} .
$$

Now, (5) and (6) together give

$$
\int_{I_{j}} g\left(\bar{x}_{j}(t)\right) d t+\int_{I_{j}} h\left(\bar{x}_{j}^{\prime}(t)\right) d t \leq \int_{I_{j}} g(\widetilde{x}(t)) d t+\int_{I_{j}} h^{* *}\left(\widetilde{x}^{\prime}(t)\right) d t .
$$

Let $\bar{x}:[0, T] \rightarrow \mathbb{R}$ be the function whose restriction to each $I_{j}(j \in \mathbb{N})$ is $\bar{x}_{j}$. 
Then $\bar{x} \in W^{1, p}$ and $\bar{x}(0)=\tilde{x}(0), \bar{x}(T)=\tilde{x}(T)$. Moreover, by (7)

$$
\begin{aligned}
\inf (\mathrm{P}) & \leq \int_{0}^{T} g(\bar{x}(t)) d t+\int_{0}^{T} h\left(\bar{x}^{\prime}(t)\right) d t \\
& \leq \int_{0}^{T} g(\tilde{x}(t)) d t+\int_{0}^{T} h^{* *}\left(\tilde{x}^{\prime}(t)\right) d t \\
& =\min (\mathrm{PR}) \leq \inf (\mathrm{P}) .
\end{aligned}
$$

It follows that the above inequalities are in fact equalities: $\bar{x}$ is a solution to (P).

Remarks. (a) The proof of Theorem 1 holds for a class of maps larger than $\mathscr{D}$. In fact, it is enough to assume that on each interval either $g^{\prime}=0$ or $g^{\prime}$ is of constant sign and that between two intervals having different signs of $g^{\prime}$, there is one with $g^{\prime}=0$.

(b) The classical example of a variational problem not having a solution is given by

$$
h\left(x^{\prime}\right)=\left|x^{\prime}-1\right|\left|x^{\prime}+1\right| \quad \text { and } \quad g(x)=x^{2}, \quad x(0)=0=x(1) .
$$

By remark (a), by modifying the function $x \mapsto x^{2}$ in an arbitrarily small neighbourhood of zero as a constant function, one obtains that the modified problem admits solutions for every choice of the boundary data.

(c) The technical tool used, Lemma 1 , is very specific to one-dimensionality; in [A-M] a counterexample to the validity of the very same lemma in higher dimension is presented. Still the authors are inclined to believe that a possibly weaker statement, e.g., density of both $g$ and $h$, should hold in general.

\section{ACKNOWLEDGMENT}

We are indebted to an unknown referee for kind remarks that improved the presentation of the paper.

\section{REFERENCES}

[A-C] M. Amar and A. Cellina, On passing to the limit for non convex variational problems, Asymptotic Anal. (to appear).

[A-M] M. Amar and C. Mariconda, A non-convex variational problem with constraints, SIAM J. Control Optim. (to appear).

[C-C] A. Cellina and G. Colombo, On a classical problem of the calculus of variations without convexity assumptions, Ann. Inst. H. Poincaré 7 (1990), 97-106.

[Ce] L. Cesari, Optimization-theory and applications, Springer-Verlag, New York, 1983.

[E-T] I. Ekeland and R. Temam, Convex analysis and variational problems, North-Holland, Amsterdam, 1977.

[M] P. Marcellini, Alcune osservazioni sull'esistenza del minimo di integrali del calcolo delle variazioni senza ipotesi di convessitá, Rend. Mat. (2) 13 (1980), 271-281.

Department of Mathematics, Sissa International School for Advanced Study, Strada Costiera 11, 34014 Trieste, Italy

E-mail address: cellina@TSMI19.SISSA.IT

Department of Mathematics, Università di Padova, via Belzoni 7, 35100 Padova, Italy

E-mail address: Mariconda@PDMAT1.UNIPD.IT 